\title{
Factores epidemiológicos y conductas de riesgo asociados al estadio sida en pacientes mayores de 15 años con infección VIH
}

\section{Epidemiological factors and risk behaviors associated with stage AIDS in patients over the age of 15 with HIV infection}

\author{
Jhonattan J.Villena-Prado,' Sonia Indacochea-Cáceda²
}

\begin{abstract}
Villena-Prado J], Indacochea-Cáceda S. Factores epidemiológicos y conductas de riesgo asociados a estadio sida en pacientes mayores de 15 años con infección VIH. Rev Soc Peru Med Interna. 2019;32(3):89-96.

https://doi.org//0.36393/spmi.v32i3.475
\end{abstract}

\begin{abstract}
RESUMEN
Овлетіvo. Determinar los factores epidemiológicos y conductas de riesgo asociados al estadio sida en pacientes mayores de 15 años. Material y Métodos. Estudio observacional, analítico y transversal. Se estudiaron 487 pacientes con infección por VIH, de los cuales 64 presentaban estadio sida, atendidos en el programa VIH-sida del Hospital Nacional Guillermo Almenara Irigoyen, en el periodo 2016-2017. Se analizaron variables epidemiológicas y conductas de riesgo. Se determinó, mediante la prueba estadística de ji cuadrado, aplicando corrección de continuidad y odds ratio la asociación entre las variables de exposición y la progresión a estadio sida. Resultados. Se asociaron a estadio sida las variables sexo masculino $(p=0,004<0,01$, OR: 4,46$)$, no uso de preservativo $(p=0,001<0,01,0 R: 14,02)$ y más de dos parejas sexuales $(p=0,001<0,01$, OR: 12,70). Asimismo, ser heterosexual se asoció como factor protector $(p=0,001<0,01$, OR: 0,021) y se determinó que la vía de transmisión más frecuente fue la sexual con 91,78\% y como comorbilidad más frecuentemente asociada a la tuberculosis con 8,21\%. Concuusión. Los factores epidemiológicos y conductas de riesgo asociados a estadio sida fueron sexo masculino, no uso de preservativo y más de dos parejas sexuales y como factor protector, ser heterosexual.
\end{abstract}

Palabras clave. sida, factores asociados, riesgo, VIH

\section{ABSTRACT}

OвJECtIVE. To determine the epidemiological factors and risk behaviors associated with the AIDS stage in patients over 15 years of age. Methods. An observational, analytical and crosssectional study was conducted. So, 487 patients with HIV infection were studied, of which 64 in AIDS stage, treated in the HIV Program of the Guillermo Almenara Irigoyen National Hospital, Lima, during the 2016-2017 period. Epidemiological variables and risk behaviors were analyzed. The association between exposure variables and progression to AIDS stage was determined using the chi-square statistical test applying continuity correction and odds ratio. Results. Among the variables studied, the following were associated with AIDS stage: male sex $(p=0,004<0,01$, OR: 4,46$)$, no condom use $(p=0,001<0,01$, OR: 14,02 having more than

I. Médico cirujano. Universidad Ricardo Palma, Lima.

2. Médica internista. Universidad Ricardo Palma. Hospital Nacional EsSalud Edgardo Rebagliati Martins, Lima. two sexual partners $(p=0,001<0,01 ;$ OR: 12,70$)$; besides, being heterosexual was associated as a protective factor $(p=$ $0,001<0,01 ;$ OR: 0,021) and it was determined that the most frequent transmission route was sexual with $91,78 \%$ and as comorbidity most frequently associated with tuberculosis with $8,21 \%$ Conclusion. The epidemiological factors and risk behaviors associated with AIDS stage were: male sex, no condom use, having more than two partners sexual and as a protective factor being heterosexual.

KEYWORDS. AIDS stage, associated factors, HIV,

\section{INTRODUCCIÓN}

La infección por el virus de la inmunodeficiencia humana (VIH) causante del síndrome de inmunodeficiencia adquirida (sida) continúa cobrando víctimas a nivel mundial. Si bien está aumentando su prevalencia de forma global, su incidencia y muertes relacionadas han disminuido porque las personas en terapia antirretroviral han extendido su tiempo de vida. 
De tal manera que sigue siendo considerado como uno de los principales problemas de salud pública a nivel mundial, y se enmarca en segundo lugar dentro de las Prioridades Nacionales de Investigación en Salud con una importante morbilidad y mortalidad en la población, especialmente en gente joven. ${ }^{1}$

Más de la mitad de las nuevas infecciones por el VIH que se producen en la actualidad afectan a los adolescentes, los cuales no tienen idea de cómo se propaga el VIH/ sida ni de cómo protegerse. ${ }^{2}$ Según los cálculos de Onusida, la tasa de mortalidad relacionada con el sida entre adolescentes ha aumentado en un $50 \%$ en los últimos siete años, a pesar de haber descendido en todos los demás grupos de edad. ${ }^{2}$ En el Perú, solo la mitad de personas que viven con infección por VIH tienen un diagnóstico. ${ }^{3}$ Dado que la enfermedad se mantiene sin síntomas por mucho tiempo, muchas personas no saben que están infectadas, lo que los pone en una situación vulnerable a los adolescentes y jóvenes por un mayor riesgo de exposición debido a que el comportamiento de estos está aún en desarrollo y estos empiezan a experimentar la vida sexual y a frecuentarla.

Desde el año 1983 en que se reportó el primer caso de sida en Perú hasta el 31 de octubre de 2018 se han notificado un total de 119042 casos de infección por VIH (109 263 casos a octubre de 2017), de los cuales 42436 se encuentran en estadio sida (40 551 casos a octubre de 2017). Los casos de VIH de Lima y Callao sumados a los casos de Loreto, La Libertad, Arequipa, Ica y Lambayeque representan el $81 \%$ de todos los casos de VIH notificados en el período 2000-2018. ${ }^{4}$

Asimismo, la dirección general de Epidemiología reportó lo siguiente: 70000 personas personas viviendo con VIH/sida en 2016; epidemia concentrada (se entiende como una prevalencia en la población clave mayor de $5 \%$ y prevalencia en gestantes menor de $1 \%$ ) en hombres que tiene sexo con hombres (HSH), con una prevalencia de $12,4 \%$ versus población general (con prevalencia $0,23 \%$ ); transmisión predominantemente sexual (97\%); relación hombre/mujer de 4/1; concentración de casos en Lima y Callao $(70 \%) .{ }^{4} \mathrm{La}$ mediana de la edad de casos de sida fue de 31 años, por lo que es posible que el $50 \%$ de los casos se hayan expuesto al VIH antes de cumplir los 21 años de edad. ${ }^{4}$

Se realizó el presente estudio para determinar los factores epidemiológicos y conductas de riesgo asociados a estadio sida en pacientes mayores de 15 años.

\section{MÉTODOS}

Se realizó un estudio observacional analítico, transversal, desarrollado en el Hospital Nacional Essalud Guillermo Almenara Irigoyen, Lima. La información se recogió de la base a los datos brindada por el Sistema de Gestión Hospitalario. Los datos recolectados fueron edad, sexo, estado civil, grado de instrucción, número de compañeros sexuales (promiscuidad), opción sexual (heterosexual, homosexual -HSH, lesbiana-, bisexual), relación sexual sin preservativo, uso de drogas inyectables, alcoholismo, vía de trasmisión, comorbilidades y estadio sida.

La población de estudio estuvo conformada por el total de fichas registradas en el Sistema de Gestión Hospitalaria del hospital, en donde se tiene los datos correspondientes de los pacientes que están en el programa de VIH/sida de 2016 a 2017, que fueron un total de 487 pacientes, de los cuales, 64 pacientes estaban en estadio sida.

\section{Análisis estadístico}

La información que se recolectó se ordenó en una base de datos del programa Microsoft Excel 2010, y se trasladó a SPSS versión 22. Se realizó un análisis descriptivo de las variables con la finalidad de caracterizar a los pacientes según frecuencias y distribución de las variables a estudiar.

Luego, se realizó estadística analítica para buscar la asociación de los factores pronósticos con la supervivencia. Se realizó análisis bivariable con determinación de OR mediante tablas de contingencia, intervalo de confianza al $95 \%$ (IC95\%), con valor $\mathrm{p}<0,05$.

\section{RESULTADOS}

En la Tabla 1 se muestra la frecuencia de los posibles los factores epidemiológicos y conductas de riesgo asociados a estadio sida de los pacientes estudiados.

Se encontraron diferencias significativas entre los dos grupos al correlacionar el desarrollo de estadio sida y las variables sexo (masculino), uso de preservativo (no uso), número de compañeros sexuales (promiscuo), cuyas estimaciones de los IC95\% de los OR > 1, así como al correlacionar el desarrollo de estadio sida y la variable opción sexual (heterosexual), cuya estimación al IC95\% del OR $<1$. 
Tabla I. Factores epidemiológicos y conductas de riesgo asociados a estadio sida en pacientes mayores de 15 años en el servicio de Medicina Interna I, Hospital Nacional Guillermo Almenara Irigoyen 2016-2017.

\begin{tabular}{|c|c|c|c|c|c|c|}
\hline \multirow[t]{3}{*}{ Variable/categorías } & \multicolumn{6}{|c|}{ Estadio sida } \\
\hline & \multicolumn{2}{|c|}{ Sí } & \multicolumn{2}{|c|}{ No } & \multicolumn{2}{|c|}{ Total } \\
\hline & $\mathrm{N}$ & $\%$ & $\mathrm{~N}$ & $\%$ & $\mathrm{~N}$ & $\%$ \\
\hline \multicolumn{7}{|l|}{ - Sexo } \\
\hline - Femenino & 4 & 4,0 & 97 & 96,0 & 101 & 100,0 \\
\hline - Masculino & 60 & 15,5 & 326 & 84,5 & 386 & 100.0 \\
\hline \multicolumn{7}{|l|}{ - Edad } \\
\hline - Otro grupo & 37 & 12,1 & 269 & 87,9 & 306 & 100,0 \\
\hline - Joven & 27 & 14,9 & 154 & 85,1 & 181 & 100,0 \\
\hline \multicolumn{7}{|l|}{ - Estado civil } \\
\hline - Soltero & 29 & 12,0 & 212 & 88,0 & 241 & 100,0 \\
\hline - No soltero & 35 & 14,2 & 211 & 85,8 & 246 & 100,0 \\
\hline \multicolumn{7}{|l|}{ - Ocupación } \\
\hline - No profesional & 57 & 14,10 & 351 & 86,0 & 408 & 100,0 \\
\hline - Profesional & 7 & 8,9 & 72 & 91,1 & 79 & 100,0 \\
\hline \multicolumn{7}{|l|}{ - Grado de instrucción } \\
\hline - No superior & 57 & $|4|$, & 346 & 85,9 & 403 & 100,0 \\
\hline - Superior & 7 & 8,3 & 77 & 91,7 & 84 & 100,0 \\
\hline \multicolumn{7}{|l|}{ - Uso de preservativo } \\
\hline - Sí & I & I,3 & 77 & 91,7 & 84 & 100,0 \\
\hline - No & 63 & 15,4 & 346 & 84,6 & 409 & 100,0 \\
\hline \multicolumn{7}{|l|}{ - Opción sexual } \\
\hline - Bisexual & 14 & 36,8 & 24 & 63,2 & 38 & 100,0 \\
\hline - Heterosexual & 2 & 8 & 254 & 99,2 & 256 & 100,0 \\
\hline - Homosexual & 48 & 24,9 & 145 & 75,1 & 193 & 100,0 \\
\hline \multicolumn{7}{|c|}{ - Número compañeros sexuales } \\
\hline - No promiscuos & 1 & I,4 & 71 & 98,6 & 72 & 100,0 \\
\hline - Promiscuos & 63 & 15,2 & 352 & 84,8 & 415 & 100,0 \\
\hline \multicolumn{7}{|c|}{ - Usuario de drogas inyectables } \\
\hline - Sí & 10 & 18,2 & 45 & 81,8 & 55 & 100,0 \\
\hline - No & 54 & 1,5 & 378 & 87,5 & 432 & 100,0 \\
\hline \multicolumn{7}{|l|}{ - Alcoholismo } \\
\hline - Sí & 10 & $|4|$, & 61 & 85,9 & 71 & 100,0 \\
\hline - No & 54 & 13,0 & 362 & 87,0 & 416 & 100,0 \\
\hline \multicolumn{7}{|l|}{ - Comorbilidad } \\
\hline - Con comorbilidad & 42 & 38,9 & 66 & 61,1 & 108 & 100,0 \\
\hline - Sin comorbilidad & 22 & 5,8 & 357 & 94,2 & 379 & 100,0 \\
\hline
\end{tabular}

Por otro lado, no se encontró diferencias significativas entre los dos grupos al correlacionar el desarrollo de estadio sida y las variables grupo de edad (joven), estado civil (soltero), ocupación (no profesional), grado de instrucción (superior), usuario de drogas inyectables, ser alcohólico (Tabla 2).

Del total de casos del sexo masculino, $15,5 \%$ (60 participantes) estaban en estadio sida, mientras que solo 4,0\% (4 participantes) del total de pacientes del sexo femenino presentaron el estadio sida.
Respecto al no uso de preservativo en relación al grado de instrucción, el mayor porcentaje de pacientes que no usaban preservativo $(87,56 \% ; n=359)$ tuvieron un grado de instrucción secundaria, seguido del grado de instrucción primaria $(9,51 \% ; n=39)$ (Figura 1).

Del $100 \%$ de participantes (72) con la condición de no promiscuo, solo 1,4\% (1 participante) presentó estadio sida. Sin embargo, en el grupo de promiscuos (415 participantes), 15,2\% (63 participantes) presentaron el estadio sida. 
Tabla 2. Factores epidemiológicos y conductas de riesgo asociados a estadio sida en pacientes mayores de 15 años en el servicio de Medicina Interna I, Hospital Nacional Guillermo Almenara Irigoyen, 2016-2017

\begin{tabular}{|c|c|c|c|c|}
\hline \multirow[t]{2}{*}{ Variable } & \multicolumn{2}{|c|}{ Estadio sida } & \multirow{2}{*}{$\begin{array}{c}\text { Odds ratio (OR) } \\
\text { (indice de confianza al 95\%) }\end{array}$} & \multirow[t]{2}{*}{$\mathrm{p}$} \\
\hline & $\begin{array}{c}\text { Si }(\%) \\
n=64\end{array}$ & $\begin{array}{l}\text { No }(\%) \\
n=423\end{array}$ & & \\
\hline - Sexo masculino & 93,8 & 77,1 & $4,463(1,582-12,592)$ & 0,004 \\
\hline - Grupo de edad (joven) & 42,2 & 36,4 & $1,275(0,747-2,175)$ & 0,451 \\
\hline - Estado civil (soltero) & 45,3 & 50,1 & $0,825(0,486-1,398)$ & 0,560 \\
\hline - Ocupación (no profesional) & 89,1 & 81,8 & $1,812(0,796-4,126)$ & 0,513 \\
\hline - Uso de preservativo (no) & 98,4 & 81,8 & $14,020(1.915-102,65 \mid))$ & 0,001 \\
\hline - Opción sexual (heterosexual) & 3,1 & 60,0 & $0,021(0,005-0,089)$ & 0,000 \\
\hline - Número de compañeros sexuales (promiscuo) & 98,4 & 83,2 & $12,707(\mid, 734-93,133)$ & 0,003 \\
\hline - Usuario de drogas inyectables & 15,6 & 10,6 & I,556 $(0,74 \mid-3,267)$ & 0,336 \\
\hline - Alcoholismo & 15,6 & 14,4 & I,099 $(0,53 \mathrm{I}-2,274)$ & 0,949 \\
\hline
\end{tabular}

Del $100 \%$ de participantes (256) con opción sexual heterosexual, solo $0,8 \%$ (2) presentaron estadio sida; $y$, en el grupo de bisexuales y homosexuales el porcentaje fue de $36,8 \%$ y $24,9 \%$, respectivamente.

Con respecto a las comorbilidades, 77,82\% $(n=379)$ de los pacientes no presentaron ninguna comorbilidad; $8,21 \%(\mathrm{n}=40)$ tenían tuberculosis (TB); 6,77\% $(\mathrm{n}=33), \quad$ sífilis; $3,25 \% \quad(\mathrm{n}=16), \quad$ condilomas acuminados; $2,25 \%(\mathrm{n}=11)$, gonorrea; $1,67 \%(\mathrm{n}=8)$ y herpes genital (Figura 2).

Con respecto a la vía de trasmisión, en 91,78\% $(n=447)$ fue por vía sexual, $6,16 \%(\mathrm{n}=30)$ por vía sanguínea y $2,06 \%(n=10)$ por vía perinatal.

\section{DISCUSIÓN}

El presente estudio fue realizado en un hospital de referencia a nivel nacional, por lo que puede dar algunas luces de cómo esta la problemática a este nivel.

Predominó el sexo masculino $(79,2 \% ; n=386)$, de los cuales 60 pacientes estaban en estadio sida, frente al sexo femenino $(20,8 \% ; n=101)$, de los cuales 4 pacientes estaban en estadio sida. La razón H/M fue 3,8, que mostró una asociación estadísticamente significativa con respecto al sexo masculino. Según el Análisis de la Situación Epidemiológica en el Perú, los casos notificados según sexo y razón H/M es similar a la encontrada en el presente estudio. ${ }^{4}$ Así, en 2018 se notificaron 3834

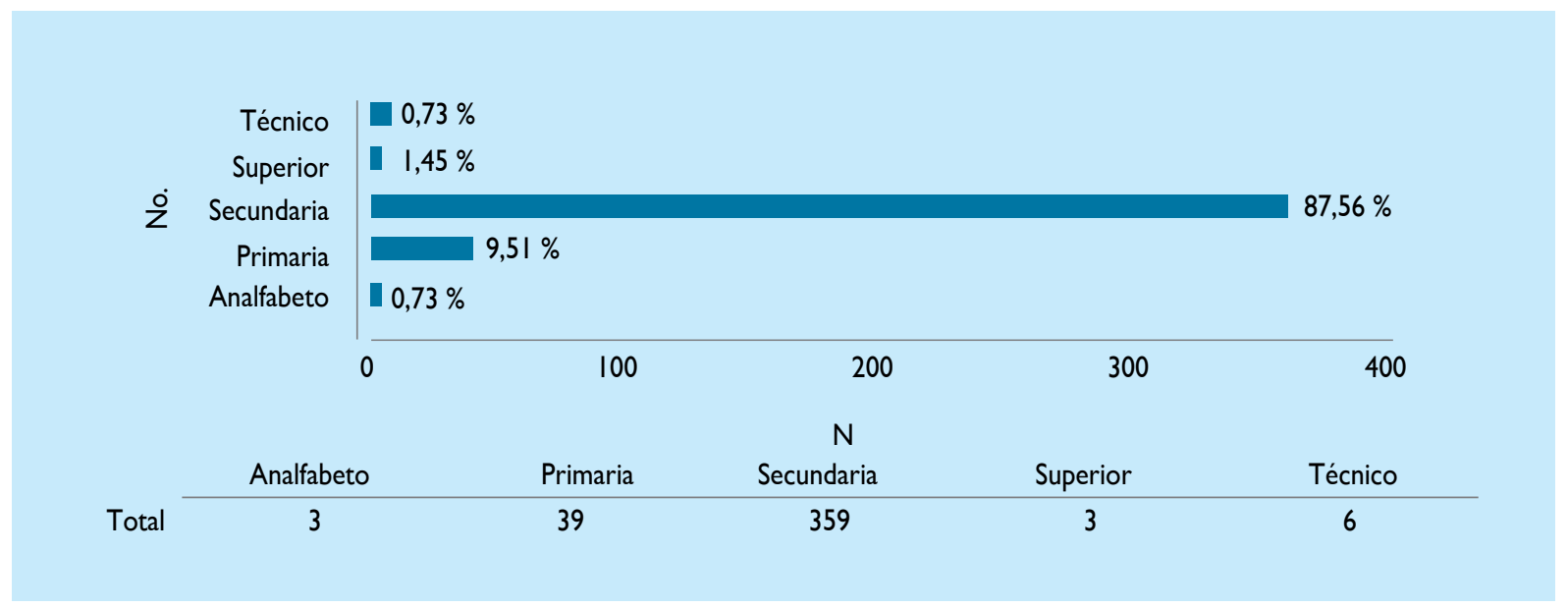

Figura I. Distribución de acuerdo al grado de instrucción vs. el no uso de preservativo en pacientes con diagnóstico de VIH, servicio de Medicina Interna, Hospital Nacional Guillermo Almenara Irigoyen, 2016-20I7. 
$(78,8 \%)$ casos de varones infectados con VIH frente a $1028(21,2 \%)$ casos de mujeres infectadas con VIH con una razón de 3,7. Esto va respaldado con los resultados de otros estudios que hallaron un predominio de varones del $66,7 \%, 80,0 \%$ y $91 \%$ de pacientes infectados con VIH..$^{5-7}$ En lo que respecta a progresión a estadio sida, algunos hallaron que el sexo masculino se asoció significativamente a un diagnóstico tardío y estadio avanzado de infección por VIH. ${ }^{8,9}$ Esto es contrario a otro estudio que identificó al sexo femenino como factor asociado a progresión rápida a sida. ${ }^{10}$

En el grupo etario se realizó la división según la clasificación de la OMS para determinar si el ser joven sería un factor que se asocie a la progresión a estadio sida. Por lo general, este grupo tiene mayores conductas de riesgo y debe ser considerado un grupo vulnerable; sin embargo, el resultado no fue significativo estadísticamente. Esto puede explicarse porque el grupo predominante en la población estudiada fue el comprendido de 30 a 59 años de edad con $59,46 \%$ $(\mathrm{n}=275)$, seguido del grupo de 18 a 29 años (jóvenes) con 37,16\% ( $n=181)$, de los cuales 27 estaban en estadio sida. Debe tenerse en cuenta que el estudio se realizó en un hospital de la seguridad social, por lo tanto, son pacientes dentro del grupo de PEA (población económicamente activa), laboralmente más estable, lo que explicaría el predominio del grupo comprendido de 30-59 años (adultos). Asimismo se debe tener en cuenta que en Perú la mediana de la edad de casos de sida es de 31 años, por lo tanto se puede deducir que, en su mayoría, se infectan cuando son adolescentes-jóvenes y progresan a estadio sida cuando ya son adultos, y que este grupo de adultos tenía una progresión más rápida a estadios avanzados de infección por VIH..$^{11}$ Otro estudio reveló que el grupo etario de 41 a 60 años se asoció a un diagnóstico tardío y estadio avanzado de infección por VIH. ${ }^{8}$

Estos resultados difieren de los dados según el Análisis de la Situación Epidemiológica en el Perú, ${ }^{4}$ que en el último quinquenio (2014-2018) observó un incremento en los casos de infección de VIH en el grupo de 20 a 29 años (población joven). ${ }^{5-7}$

Con respecto al estado civil, 49,48\% $(n=241)$ de infectados con VIH eran solteros, similar a otro estudio que encontró $58,59 \%$ de solteros en los infectados con VIH. En contraste, otro estudio halló que 54,2\% de oficiales infectados con VIH eran casados. ${ }^{12,13}$ Las personas solteras tienden a tener una vida sexual más libertina y mayor número de parejas sexuales porque tienen mayor autonomía e independencia en comparación con otros tipos de estado civil. ${ }^{14}$

Con respecto al grado de instrucción y la ocupación profesional, en el presente estudio no se encontró asociación. Sin embargo, algunos estudios indican que el poseer un mayor nivel educativo (grado de instrucción superior) y el ser profesional estaba asociado estadísticamente en forma significativa como factor protector para progresión a estadio sida. ${ }^{9,11}$ Es de esperarse que los pacientes al recibir una educación de tipo universitaria tengan mayor conciencia y responsabilidad en cuanto al cuidado y la prevención de las infecciones de transmisión sexual (ITS). Un estudio, en Huánuco, halló que $60 \%$ de los pacientes tenían grado de instrucción secundaria y solo $10 \%$, grado de instrucción superior. ${ }^{15}$ Esto podría estar relacionado a que poseer un grado de instrucción superior denotaría una mayor reserva cognitiva y por lo tanto tener una mejor compresión de la enfermedad y de cómo prevenirla, pero también se debe considerar que en la sociedad peruana no todos acceden a la educación superior.

Acerca del número de parejas sexuales, se subdividió en dos grupos: pacientes promiscuos y no promiscuos (la definición de promiscuo incluye a aquellos pacientes que tuvieron más de dos parejas sexuales, según la OMS), siendo un $85,43 \%(n=416)$. Un estudio encontró que $72,5 \%$ de mujeres embarazadas con VIH habían tenido dos a tres parejas sexuales; otro, que $74 \%$ habían tenido dos o más parejas sexuales luego de saber que tenían el diagnóstico de VIH. ${ }^{16,17}$ En cuanto a la progresión de estadio sida, un estudio ${ }^{10}$ obtuvo que el tener más de cinco parejas sexuales se asoció como factor de riesgo para progresión rápida a estadio sida, pero de forma no significativa estadísticamente, lo que sí se halló en el presente estudio.

En cuanto a la opción sexual, $52,56 \%(\mathrm{n}=256)$ fueron heterosexuales y $36,63 \% \quad(n=193)$, homosexuales, resultado similar a otros estudios donde el mayor porcentaje fueron pacientes heterosexuales promiscuos. ${ }^{17,18}$ En contraste, otros estudios encontraron que la opción sexual predominante de los pacientes infectados por VIH fue de homosexuales. ${ }^{13,19}$ En cuanto a la progresión a estadio sida, un estudio concluyó de forma estadísticamente significativa que el grupo de heterosexuales tuvo menor frecuencia de enfermedad avanzada por VIH comparado con el grupo de 
bisexuales, quienes fueron el grupo predominante para diagnóstico tardío y enfermedad avanzada por VIH. ${ }^{8}$ De manera similar, el presente estudio halló que solo $0,8 \%$ de los heterosexuales presentaron estadio sida, estadísticamente significativo como factor protector.

En cuanto al uso de preservativos, 84,18\% $(\mathrm{n}=410)$ no lo usaban vs. $15,82 \%(\mathrm{n}=77)$ que sí lo usaban. Asimismo, predominó el grado de instrucción secundaria en los pacientes que no usaban preservativo con $87,56 \%(\mathrm{n}=359)$. El INEI realizó una encuesta en mujeres de 15 a 49 años de edad que usaron condón en la última relación sexual en los últimos tres meses, según área de residencia y nivel de educación. Se encontró que, en el año 2017, solo 14,1\% de las mujeres a nivel nacional usaron condón en su última relación sexual, con una mejoría respecto a 2014, que fue $12 \% .^{20}$ En la misma encuesta, se comparó el uso de condón en relación al grado de instrucción, en el año 2017 se obtuvo que usaron condón 5,6\% de mujeres con primaria, $13,7 \%$ con secundaria y $19,2 \%$ con nivel superior. Esto se debe evaluar en el contexto en el que la OMS pone como primera medida para la prevención de la infección por VIH el uso de preservativo. ${ }^{21}$ Estos resultados siguen siendo preocupantes, pues exponen a contraer ITS y embarazos no deseados.

Otros estudios mostraron resultados similares, donde las mujeres que no usaban preservativo tuvieron un riesgo moderado-alto de transmisión de VIH y donde 85,4\% tenían relaciones sexuales sin condón a pesar de conocer su diagnóstico y, peor aún, al 36,6\% le daba igual no protegerse porque ya tenía VIH. ${ }^{17,22}$ En contraste, en España, un estudio concluyó que "los pacientes desde que fueron diagnosticados tomaron mayor conciencia sobre las vías de transmisión y tuvieron una sexualidad más protegida que previamente al diagnóstico". 23 En cuanto a la progresión a estadio sida, un estudio concluyó que el no uso de condón o preservativo actuaba como un factor asociado a progresión a estadio sida de forma estadísticamente significativa, similar al resultado encontrado en el presente estudio. ${ }^{10}$

En lo referente a los pacientes usuarios de drogas y la progresión a estadio sida, no se encontró asociación significativa en el presente estudio como sí en otro donde los pacientes drogadictos infectados con VIH tenían un diagnóstico tardío y una presentación de enfermedad avanzada, lo que también los exponían a una mayor morbimortalidad. ${ }^{8}$
En cuanto al alcoholismo, se encontró en el presente estudio que $85,42 \%(n=416)$ no eran alcohólicos, en contraste con otros donde hasta $50 \%$ de pacientes infectados tenían como hábito nocivo el alcoholismo. ${ }^{5,18}$ En relación a la progresión a estadio sida, no se halló en este estudio una asociación de forma significativa, en contraste con otro estudio que encontró que los pacientes infectados y con consumo elevado de alcohol presentaban un estadio clínico avanzado de infección por VIH.

En lo que respecta a la presencia de comorbilidades en pacientes con diagnóstico de VIH, se encontró que el $77,82 \%(\mathrm{n}=379)$ de los pacientes no presentó alguna complicación asociada, 8,21\% $(\mathrm{n}=40)$ tenía TB y el resto de pacientes $(13,97 \%)$, ITS. (Figura 2).

Esto podría ser reflejo al mayor acceso del tratamiento antirretroviral (TAR) en los establecimientos de salud, puesto que según la Dirección General de Intervenciones Estratégicas en Salud Pública para el año 2017 se tenían un total de 147 establecimientos de salud que brindaban TAR, con la consecuente menor carga viral y aumento de CD4, para evitar, así, la aparición de enfermedades oportunistas. ${ }^{4}$

En el estudio en el Hospital Rebagliati, encontró como comorbilidad principal a la TB con $20,2 \%$ y otro en España ${ }^{23}$ encontró a la TB (pulmonar y extrapulmonar) como enfermedad concomitante más frecuente..$^{18,23} \mathrm{En}$ nuestro medio, la alta prevalencia de TB asociada a la inmunodeficiencia producida por el VIH reflejaría el porcentaje relativamente alto del presente estudio.

En cuanto a las ITS, algunos estudios tuvieron como comorbilidad más frecuente la sífilis $(7,25 \%$ y $11,8 \%)$ y otro estudio encontró a la condilomatosis como la principal comorbilidad asociada al VIH., ${ }^{524-26}$ Esto es reflejo de la forma de contagio sexual y del no uso de preservativo.

Finalmente, con respecto a la vía de transmisión, se obtuvo que $91,78 \%(\mathrm{n}=447)$ se infectaron por vía sexual; $6,16 \%(n=30)$, por vía sanguínea; $2,06 \%$, $(\mathrm{n}=10)$ por vía perinatal. Según el Análisis de la Situación Epidemiológica en el Perú, entre los años 2014-2018, 98,9\% se contagiaron por vía sexual; $0,7 \%$, por vía parenteral; $0,3 \%$, por vía vertical ${ }^{4}$ Cabe destacar la disminución de la vía de contagio vertical, debido a que la norma técnica en las gestantes con VIH es que tengan una carga viral indetectable para disminuir la probabilidad de contagio. 


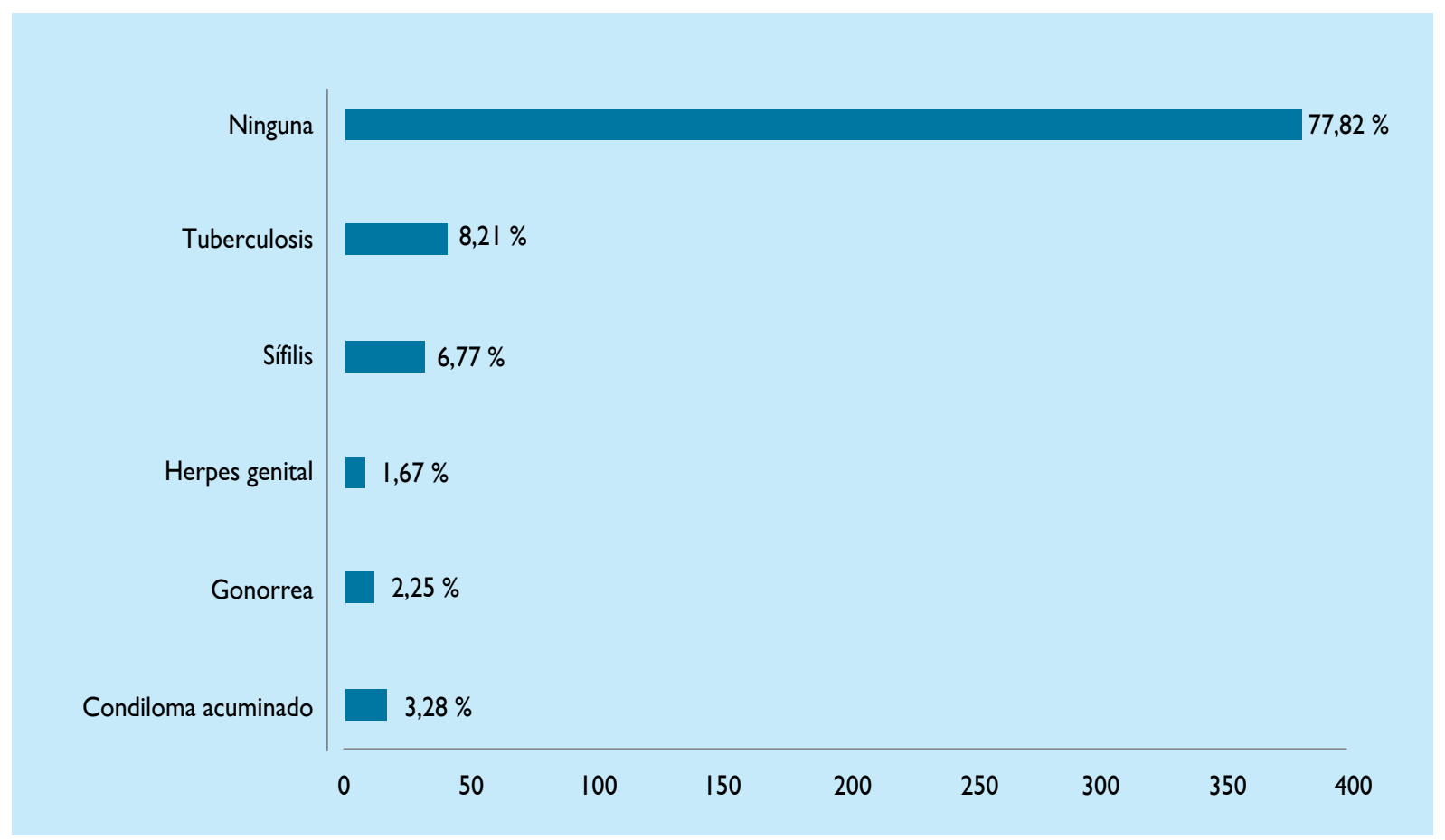

Figura 2. Comorbilidades en pacientes con diagnóstico de VIH, servicio de Medicina Interna, Hospital Nacional Guillermo Almenara Irigoyen, 2016.

La mayoría de autores concuerda que la vía de transmisión más frecuentemente asociada a la infección por VIH es la sexual..$^{13,24,27}$ Sin embargo, un estudio realizado en Irán mostró que el uso de drogas inyectables fue la más frecuente, con $59 \%$, seguida de la vía sexual con $13 \%$.

En conclusión, los factores epidemiológicos en pacientes con infección por VIH que se asociaron a estadio sida de forma estadísticamente significativa en el presente estudio fueron el sexo masculino y tener una opción sexual distinta a la heterosexual (homosexual, bisexual).

Las conductas de riesgo que se asociaron a infección por VIH y estadio sida de forma estadísticamente significativa fueron el número de compañeros sexuales (pacientes promiscuos con dos o más compañeros) y el no uso de preservativo durante el mantenimiento de relaciones sexuales.

La forma de transmisión más frecuente en la población estudiada fue la vía sexual y la comorbilidad más frecuentemente asociada, la tuberculosis.

\section{REFERENCIAS BIBLIOGRÁFICAS}

I. Maartens G, Celum C, Lewin SR. HIV infection: epidemiology, pathogenesis, treatment, and prevention. Lancet. 20I4;384(9939):258-7I.

2. Who's Certified [Internet] Onusida no se olvida de los adolescentes que viven con el $\mathrm{VIH}$. [citado 6 de mayo de 2019]. URL disponible en: http://www.unaids.org/es/resources/presscentre/featurestories/2014/ april/20I40430adolescents

3. Who's Certified [Internet] | Perú Onusida. [citado 6 de mayo de 2019]. URL disponible en: http://www.unaids.org/es/regionscountries/ countries/peru

4. Centro Nacional de Epidemiología, Prevención y Control de Enfermedades. Situación epidemiológica del VIH-sida en el Perú [Internet]. Perú: Ministerio de Salud; 2018 oct p. I5. URL disponible en: http://www.dge.gob.pe/portal/docs/vigilancia/vih/Boletin_2018/octubre. pdf

5. Bonilla-Ruiz AM, Vilcapoma-Balbín P. Las características clínicas, epidemiológicas e inmunoserológicas de los pacientes en terapia antiretroviral de gran actividad en un hospital peruano. Rev Médica Panacea. 2013;3(I):19-23. URL disponible en: http://revpanacea.unica. edu.pe/index.php/RMP/article/view/99

6. Valdés Fuster JL, Oliva Venereo DC,Viñas MartínezAL, Lastre Hernández D, Camilo Cuéllar YA. Características clínico-epidemiológicas de los pacientes con el virus de inmunodeficiencia humana. Boyeros. 2013. Rev haban cienc méd. [Internet]. 2016;15(6):955-967. [citado 2019 May 7] URL disponible en: http://scielo.sld.cu/scielo.php?script=sci_ arttext\&pid=S1729-519X20160006000I I\&Ing=es.

7. Haghgoo SM, Joula H, Mohammadzadeh R, Sabour S, Yousefi R, Ghahramani G, et al. Epidemiology of HIV/AIDS in the East Azerbaijan Province, Northwest of Iran. Jundishapur J Microbiol. 2015;8(8):el 9766. 
8. Maquera-Afaray J, Cvetkovic-Vega A, Cárdenas MM, Kälviäinen H, Mejia CR. Diagnóstico tardío y enfermedad avanzada de VIH en pacientes adultos en un hospital de la seguridad social de Perú. Rev Chil Infectol. 2016;33:20-6.

9. Warley E, Fernández Galimberti G, Vieni MI, Tavella S, Salas M, Desse J, et al. Factores asociados al estadio clínico avanzado en el inicio de la terapia antirretroviral. Medicina (B.Aires) [Internet]. 20I2:72(5):367-370. [citado 2019 May 7] URL disponible en: http://www.scielo.org.ar/scielo. php?script=sci_arttext\&pid=S0025-768020 I $2000600002 \& \operatorname{lng}=e s$

10. Abrahantes Rodríguez Y, Pérez Ávila J, Kourí Cardellá V, Fonseca Gómez C, Baly Gil A, Tápanes Fernández T. Factores asociados a la progresión rápida a sida en individuos cubanos. Rev Cub Med Trop. 2010;62(2): I4653.

II. Monge S, Romero J, Rodríguez C, Mendoza M, Górgolas M, Cosin J, Dronda $F$, et al. Factores sociodemográficos asociados a la progresión de la infección por VIH e impacto del TARGA en una cohorte de pacientes seroconvertores en Madrid (1986-2009). Science Direct. 2012; 30(3):II7-23. Disponible en: https://www.sciencedirect.com/science/ article/pii/S02 I3005XII00263I

12. Sánchez-Naranjo H. Características clínicas y epidemiológicas de la infección por $\mathrm{VIH} /$ sida en el municipio Yara en el período 1986-20I4. Multimed [en Internet]. 2017 [citado 2019 May 7]; 19(3):[aprox. 0 p.].URL disponible en: www.revmultimed.sld.cu/index.php/mtm/article/view/344

13. Palomino Rosas, Sherlly F. Características epidemiológicas de las personas que viven con VIH/sida atendidas en el Programa VIH/sida del servicio de Enfermedades Infecciosas del Hospital Nacional PNP "Luis N. Sáenz" 1988-2010. Univ Nac Mayor San Marcos [Internet]. 20I I [citado 6 de mayo de 2019]. URL disponible en: http://cybertesis.unmsm.edu.pe/ handle/cybertesis//862

14. Who's Certified [Internet] OMS | Desarrollo en la adolescencia.WHO. [citado 6 de mayo de 2019]. URL disponible en: http://www.who.int/ maternal_child_adolescent/topics/adolescence/dev/es/

15. Bejarano F, Chanamé J, Dámaso B, Palacios E. (Instituto Nacional de Salud) Factores de la adherencia de las personas que viven con $\mathrm{VIH} /$ sida que reciben esquema TARGA en el Hospital Regional Hermilio Valdizán Huánuco [Internet]; 2006 p. 82. URL disponible en: https://bvs.ins.gob.pe/ insprint/CINDOC/INFORMES_TECNICOS/75.pdf

16. Rivera Videz M, Darío Fernández R.Asociación de condilomatosis y VIH en mujeres embarazadas en el Hospital Materno Infantil durante el período de septiembre 2002 a julio 2004. Rev Méd Postgr Med. 2006; 9(2):5.

17. Palomino Valencia C. Características de la conducta sexual en pacientes diagnosticados con VIH en el Hospital Nacional Arzobispo Loayza durante el año 2016 [Internet] [Tesis pre-grado]. [Lima-Perú]: Universidad Privada San Juan Bautista; 2018. URL disponible en: http://repositorio. upsjb.edu.pe/bitstream/handle/upsjb/ I 645/T-TPMC-\%20Claudia\%20 Gabriela\%20\%20Palomino\%20Valencia.pdf?sequence= I \&isAllowed=y

18. Olivares Nunura J. Factores clínicos y epidemiológicos más frecuentes de los pacientes con diagnóstico de $\mathrm{VIH} /$ sida atendidos en el Hospital de Apoyo Nuestra Señora de las Mercedes, Paita durante el periodo julio 2016-junio 2017. [Internet]. [Piura]: Universidad Nacional de Piura; 2018. URL disponible en: http://repositorio.unp.edu.pe/handle/UNP/II9I.
19. Narváez Guerra O. Características clínicas y epidemiológicas de la infección por $\mathrm{VIH}$ en pacientes mayores de 50 años atendidos en el Hospital Nacional Edgardo Rebagliati Martins, Lima 2008-20I 2 [Internet]. [Arequipa]: Universidad Católica de Santa María; 2014. Disponible en: http://tesis.ucsm.edu.pe/repositorio/handle/UCSM/4895

20. INEI. Perú, Enfermedades no transmisibles y transmisibles, 2017 [Internet]. Lima: Instituto Nacional de Estadística e Informática; 2018. URL disponible en: https:/www.inei.gob.pe/media/MenuRecursivo/ publicaciones_digitales/Est/Lib I526/index.html

21. Who's Certified [Internet] VIH/SIDA [Internet]. [citado 6 de mayo de 2019]. Disponible en: https://www.who.int/es/news-room/fact-sheets/ detail/hiv-aids

22. Ramos W, Munive L, Calderón M, Velazco S, Velazco M, Características sociodemográficas y fuentes de información en relación con la percepción del riesgo de transmisión delVIH y sida de mujeres peruanas en edad fértil. Período 2004-2006. Revista Peruana de Epidemiología. [Internet] 2009;13(3):I-7. URL disponible en: https://www.redalyc. org/articulo.oa?id=203 | 20367007 ">http://portal.amelica.org/ameli/ jatsRepo/203 I 20367007</a>

23. Villar Salgueiro M, Fernández IL.Vivencia de la sexualidad de las personas con VIH. Univ Almería. [Internet] 2012;76. Disponible en: http://www. sidastudi.org/resources/inmagic-img/DD 16082.pdf

24. Grandal FM. Características clínicas, virológicas e inmunológicas de los nuevos diagnósticos de infección por $\mathrm{VIH}$ en el área sanitaria de $\mathrm{A}$ Coruña en los últimos 10 años evolución clínica y respuesta al tratamiento antirretroviral. [Internet] Universidad de La Coruña 20|4:60. URL disponible en: http://hdl.handle.net/2/83//2420

25. Montúfar AF, Quiroga A, Builes C, Saldarriaga C, Aguilar C, Mesa M, Zuleta TJ. Epidemiología de la infección por el virus de inmunodeficiencia humana en pacientes hospitalizados en una institución de alta complejidad y enseñanza universitaria en Medellín, Colombia. Science Direct. 2016; 20(I): 9-16. URL disponible en: https://www.sciencedirect. com/science/article/pii/SOI23939215000776

26. Sandoval EA, Chavarría YY, Bustillo KR. Características clínicas y epidemiológicas de los pacientes adultos con $\mathrm{VIH}$ en el Instituto Hondureño de seguridad social. Rev Med Hondur. 2009;77(4):I65-169. URL disponible en: http://www.bvs.hn/RMH/pdf/2009/pdf/Vol77-4-20094.pdf

27. Lancheros Silva AM. Caracterización epidemiológica del VIH-sida en el departamento de Boyacá desde 2010 a 2012. Universidad Nacional de Colombia. [Internet] 20|4:76. URL disponible en: http://bdigital.unal.edu. $\mathrm{co} / 43 \mathrm{I} 2 \mathrm{I}$

CORRESPONDENCIA:Jhonattan J.Villena-Prado jhonv1807@gmail.com

CONFLICTO DE INTERÉs: Los autores declaran no tener conflicto de interés alguno. FINANCIAMIENTO: Financiado por los autores.

FECHA DE RECEPCIÓN: 6 de octubre de 2019.

FECHA DE ACEPTACIÓN: 20 de octubre de 2019. 